Chinese Journal of Organic Chemistry

ARTICLE

\title{
碳负载铜一锅法催化合成苯并硒唑类衍生物
}

\author{
周美云 ${ }^{a}$ 吴照钧 ${ }^{a}$ 李毅群 ${ }^{a}$ 郑文杰 ${ }^{a}$ 纪盛滨 ${ }^{a}$ 周 华*,b \\ $\left({ }^{a}\right.$ 暨南大学化学系 广州 510632)

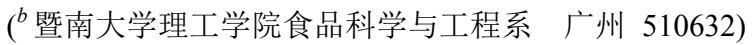

\begin{abstract}
摘要 建立了一种简单实用、经济高效的以邻溴苯胺为起始原料, 廉价的活性碳负载铜为催化剂, 在室温条件下一锅 法合成苯并硒唑类衍生物的方法，得到了中等到优良的产率. 本方法具有催化剂廉价易得、操作简便、合成效率高的 优点.

关键词 一锅法合成; 苯并硒唑; 碳负载铜催化
\end{abstract}

\section{One-Pot Synthesis of Benzo[d][1,3]selenazole Derivatives by Using Carbon-Supported Copper Catalyst}

\author{
Zhou, Meiyun $^{a}$ \\ $\mathrm{Wu}$, Zhaojun $^{a}$ \\ Li, Yiqun ${ }^{a}$ \\ Zheng, Wenjie ${ }^{a}$ \\ $\mathrm{Ji}$, Shengbin ${ }^{a}$ \\ Zhou, Hua*,b \\ ( ${ }^{a}$ Department of Chemistry, Jinan University, Guangzhou 510632) \\ ( ${ }^{b}$ Department of Food Science and Engineering, Jinan University, Guangzhou 510632)
}

\begin{abstract}
A simple, efficient, inexpensive one-pot method for the benzo[ $d][1,3]$ selenazole derivatives synthesized from 2-bromoaniline was developed by using cheap carbon-supported copper as catalyst at room temperature. The reaction provides an efficient protocol to a series of benzo $[d][1,3]$ selenazole derivatives in good to high yields, and benefits of readily available catalyst and synthesis of high efficiency.
\end{abstract}

Keywords one-pot synthesis; benzo[ $[d][1,3]$ selenazole; carbon-supported copper

含硒化合物因为其广泛的生物活性, 引起了科学家 们的强烈兴趣 ${ }^{[1]}$. Bayoumy 等 ${ }^{[2]}$ 证实, 某些合成的有机硒 化物比天然硒化物有着更强的生物活性, 而毒性则更 低. 例如, 硒原子直接与芳环相连的有机硒化合物, 其 $\mathrm{C}$ - $\mathrm{Se}$ 键较其他连接方式更加稳定, 在生命代谢过程中, 硒原子难以进入硒库, 而导致其毒性下降. 苯并硒唑是 一类新型的有机硒化合物, 在药学、染料等方面表现出 潜在的应用价值 ${ }^{[3]}$. 因此对苯并硒唑类化合物的研究, 引起了科学家们的高度关注. 苯并硒唑类化合物的合成 涉及到 $\mathrm{C}-\mathrm{Se}$ 键的构建, 目前主要以铜基催化剂为主 ${ }^{[4]}$. Fujiwara 等 ${ }^{[5]}$ 报道以 $\mathrm{CuI}$ 为催化剂, 使异硒氧酸酯与胺、 醇或硫醇发生环化加成反应得到相应的产物. Sashida 等 ${ }^{[6]}$ 报道了以 $\mathrm{CuI}$ 为催化剂, 环化合成了 $\mathrm{N}$ 取代的苯并 硒唑衍生物的合成方法. Kaname 等 ${ }^{[7]}$ 以邻碘苯胺及其取
代物为原料, 以 $\mathrm{Cu}(\mathrm{OTf})_{2}$ 为催化剂, 采用一锅法合成了 系列苯并硒唑类衍生物. 总体而言, 目前苯并硒唑类衍 生物合成所使用的铜基催化剂基本以含铜化合物为主, 而负载型铜催化剂使用于该类化合物的合成很少被报 道. 工业上, 负载型铜基催化剂是一类极其重要的催化 剂, 因其良好的催化性能和相对低廉的价格在诸多重要 工业催化反应中得到广泛应用 ${ }^{[8]}$.

本文报道一种反应条件温和、以经济高效的碳负载 铜为催化剂、一锅法合成苯并硒唑类衍生物的方法. 以 邻溴苯胺为原料, 加入甲酸, 与氧化锌进行研磨, 然后 加入固体光气与单质硒反应生成相应的异硒氧化合物, 而后在碳负载纳米铜的催化下，与仲胺化合物或醇类化 合物反应生成苯并硒唑类衍生物(Scheme 1) ${ }^{[5]}$. 与文献 比较, 该过程反应更快, 处理更加简单, 且不需要分离

\footnotetext{
*E-mail: zhhua-1@163.com

Received December 12, 2014; revised January 20, 2015; published online February 10, 2015.

Project supported by the National Natural Science Foundation of China (Nos. 31101323, 31000816).

国家自然科学基金(Nos. 31101323, 31000816)资助项目.
} 
中间产物，催化剂可以反复使用，反应效率更高.
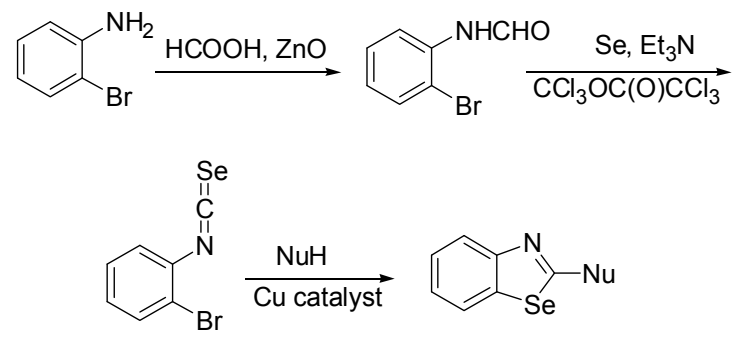

图式 1 苯并硒唑类衍生物的合成路线

Scheme 1 Synthetic pathway toward benzo[d][1,3]selenazole derivatives

\section{1 结果与讨论}

\section{1 反应条件优化}

首先以吗啡啉与邻溴异硒氧酸酯为反应模板, 系统 地研究了反应温度、反应时间、反应溶剂、碱等对反应 的影响, 结果见表 1 . 从表 1 可以看出, 反应温度对此反 应的影响较大. 通过 TLC 法跟踪和后处理发现, 反应的 最佳温度为室温, 当温度升高时, 反应的副产物增多, 产品收率降低(表 1, Entries 1 3). 反应时间对反应的影 响不明显, 反应 $1 \mathrm{~h}$ 后, 原料已基本反应完全(表 1, Entries 1, 4 5). 碱对本反应的影响较大, 1,8-二氮杂二环 十一碳-7-烯(DBU)、三乙胺(表 1, Entries 11, 12)等有机 碱时效果较好, 其他无机碱相对较差. 溶剂影响不可忽 略, 以 THF, 二氯甲烷为溶剂时, 反应时间最短, 产率 最高(表 1, Entries 1,13). 甲醇作为溶剂时, 副反应增多, 从而影响反应的产率; 乙醚和丙酮作为反应溶剂时, 不 仅副产物增多, 而且反应过程中生成大量的絮状物, 粘 附在催化剂的表面, 影响催化效果, 从而造成产率降低.

表 1 反应条件笁选与优化 ${ }^{a}$

Table 1 Optimization of reaction condition

\begin{tabular}{|c|c|c|c|c|c|}
\hline Entry & Solvent & Base & Temp. $/{ }^{\circ} \mathrm{C}$ & Time/h & Yield $^{b} / \%$ \\
\hline 1 & $\mathrm{CH}_{2} \mathrm{Cl}_{2}$ & DBU & r.t & 1 & 80 \\
\hline 2 & $\mathrm{CH}_{2} \mathrm{Cl}_{2}$ & DBU & 40 & 1 & - \\
\hline 3 & $\mathrm{CH}_{2} \mathrm{Cl}_{2}$ & DBU & Reflux & 1 & - \\
\hline 4 & $\mathrm{CH}_{2} \mathrm{Cl}_{2}$ & DBU & r.t & 2 & 80 \\
\hline 5 & $\mathrm{CH}_{2} \mathrm{Cl}_{2}$ & DBU & r.t & 3 & 80 \\
\hline 6 & $\mathrm{CH}_{2} \mathrm{Cl}_{2}$ & $\mathrm{NaOH}$ & r.t & 3 & 65 \\
\hline 7 & $\mathrm{CH}_{2} \mathrm{Cl}_{2}$ & $\mathrm{KOH}$ & r.t & 3 & 70 \\
\hline 8 & $\mathrm{CH}_{2} \mathrm{Cl}_{2}$ & $\mathrm{Na}_{2} \mathrm{CO}_{3}$ & r.t & 4 & 67 \\
\hline 9 & $\mathrm{CH}_{2} \mathrm{Cl}_{2}$ & $\mathrm{Et}_{3} \mathrm{~N}$ & r.t & 1 & 75 \\
\hline 10 & THF & DBU & r.t & 1 & 80 \\
\hline 11 & $\mathrm{CH}_{3} \mathrm{COCH}_{3}$ & DBU & r.t & 1 & 75 \\
\hline 12 & $\mathrm{C}_{2} \mathrm{H}_{5} \mathrm{OC}_{2} \mathrm{H}_{5}$ & DBU & r.t & 1 & 50 \\
\hline 13 & $\mathrm{CH}_{3} \mathrm{OH}$ & DBU & r.t & 1 & 40 \\
\hline
\end{tabular}

${ }^{a}$ Reaction conditions: formamides $(3 \mathrm{mmol})$, triethylamine $(12.8 \mathrm{mmol})$, triphosgene $(1.6 \mathrm{mmol})$, DBU $(3 \mathrm{mmol})$, secondary amine $(3.6 \mathrm{mmol})$, $\mathrm{Cu}-\mathrm{C}$ $(0.05 \mathrm{~g}) .^{b}$ Isolated yield based on aryl amines.

\section{2 活性碳负载纳米铜催化剂的评价与表征}

\subsection{1 活性碳的粒径对反应的影响}

考察了两种不同粒径(分别为 100,200 目)的活性碳 作为催化剂载体, 经催化剂活性评价后发现, 200 目的 活性碳的催化效果要稍微优于 100 目(表 2, Entries 1,2). 为了探寻两者催化剂活性不同的原因, 我们扫描了两种 载体粒径不同的催化剂的 SEM 图(图 1). 从图 1 可以看 出，在 100 目活性碳粒径上铜分布不均匀，且有团聚现 象，催化活性中心较少; 200 目的活性碳粒度更均匀，铜 在其表面上分散较好. 可能的原因在于担体颗粒较小 时，活性成分受外界因素(包括还原剂滴加速度、搅拌速 度)的影响可能相对更小，活性成分分布更容易控制.
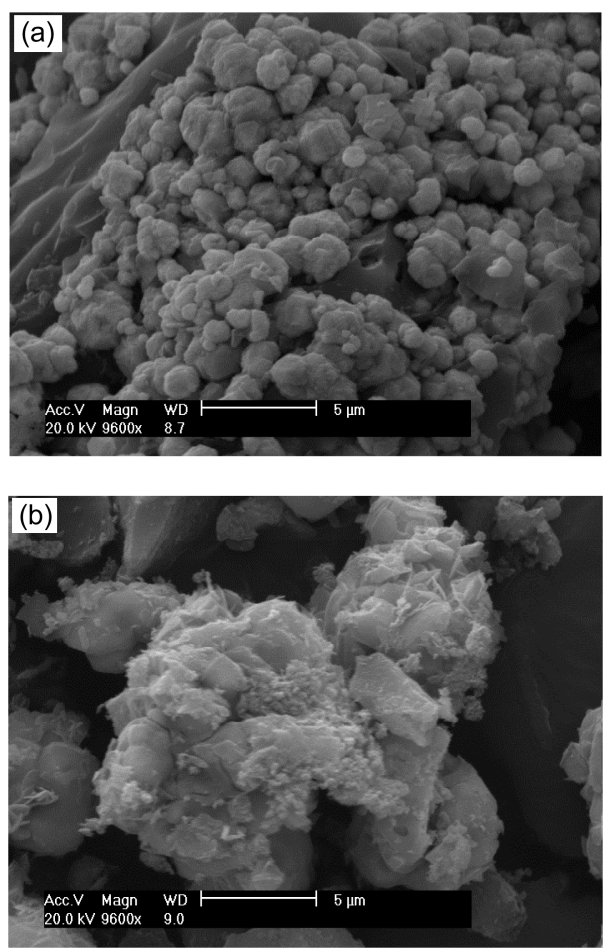

图 $1 \mathrm{Cu} / \mathrm{C}$ 催化剂的 SEM 图

Figure 1 SEM images of the catalyst $\mathrm{Cu} / \mathrm{C}$ ${ }^{a} 100$ mesh activated carbon; ${ }^{b} 200$ mesh activated carbon

\subsection{2 还原程度对反应的影响}

采用水合肼对负载纳米铜进行还原. 当滴加还原剂 水合肼的量较少时 ( $3.0 \mathrm{~mL}$ 水合肼), 铜的价态以 +2 和 +1 共存, 以 +2 价为主, 用该催化剂得到的转化率仅 38\%(表 2, Entry 1); 逐步增加还原剂水合肼的用量, 催 化剂所对应的活性逐步提高, 反应的转化率从 $38 \%$ 提升 到 84\%(表 2, Entries 1，3，4); 然而, 再进一步增加还原 剂水合肼的用量, 催化活性不升反降, 反应的转化率从 84\%降低到 44\%(表 2, Entries 4 6). 原因可能在于催化 剂中铜价态的变化: 当滴加还原剂水合肼的量较少时, 催化剂中铜的价态以 +2 和 +1 共存, 以 +2 价为主; 随 
表 2 活性碳尺寸、还原剂用量的对催化活性影响 ${ }^{a}$

Table 2 Effect of size of active carbon, amount of reduction on catalytic activity

\begin{tabular}{ccccc}
\hline Entry & $\mathrm{Mesh} / \mathrm{C}$ & $\mathrm{C} / \mathrm{g}$ & $V\left(\mathrm{~N}_{2} \mathrm{H}_{4} \bullet \mathrm{H}_{2} \mathrm{O}\right) / \mathrm{mL}$ & $\mathrm{Yield}^{b} / \%$ \\
\hline 1 & 100 & 5.0 & 3.0 & 38 \\
2 & 200 & 5.0 & 3.0 & 48 \\
3 & 100 & 5.0 & 5.0 & 70 \\
4 & 100 & 5.0 & 10.0 & 84 \\
5 & 100 & 5.0 & 15.0 & 80 \\
6 & 100 & 5.0 & 25.0 & 44
\end{tabular}

${ }^{a}$ Reaction conditions: formamides $(3 \mathrm{mmol})$, triethylamine $(12.8 \mathrm{mmol})$, $\mathrm{CH}_{2} \mathrm{Cl}_{2}(12 \mathrm{~mL})$, triphosgene $(1.6 \mathrm{mmol})$, DBU (3 mmol), secondary amine (3.6 mmol), Cu-C (0.05 g). ${ }^{b}$ Isolated yield based on aryl amines.

着还原剂用量的增加, 催化剂铜的价态从 +2 为主演变 为以 +1 价为主; 当还原剂用量增加到一定量后，催化 剂铜的价态变成零价. 为了确认这一过程, 我们对催化 剂进行 XRD 表征. 图 2 为催化剂被部分还原 $(10.0 \mathrm{~mL}$ 水合肼)和完全还原 $(25.0 \mathrm{~mL}$ 水合肼)后的 XRD 图. 完全 还原的催化剂(25.0 mL 水合肼)与单质铜的标准衍射峰 基本吻合，说明 $\mathrm{Cu}^{2+}$ 已基本完全还原成 $\mathrm{Cu}$; 部分还原 (10.0 mL 水合肼)时催化剂基本只出现 $\mathrm{Cu}_{2} \mathrm{O}$ 峰, 说明 $\mathrm{Cu}^{2+}$ 基本完全还原成了 $\mathrm{Cu}^{+}$. 结合催化剂的评价结果, 不难得出结论: $\mathrm{Cu}^{+}$可能是催化活性中心. 该结论对进 一步优化催化剂性能具有重要的指导意义.

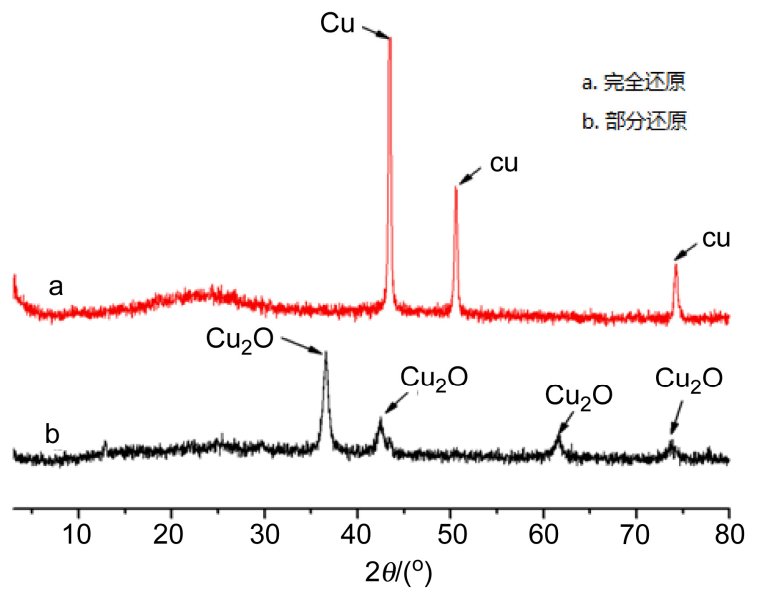

图 $2 \mathrm{Cu} / \mathrm{C}$ 催化剂的 XRD 图

Figure 2 XRD patterns of the catalyst $\mathrm{Cu} / \mathrm{C}$

\section{3 底物适用范围的研究}

获得活性碳负载铜催化合成 2-(1,4-氧氮杂环己 基)-1,3 苯并硒唑的最佳条件(表 1, Entry 13)后, 我们进 一步考察此催化体系的普适性(表 3). 从表 3 可知: 催化 体系适合多种不同取代的仲胺、苯酚和苯硫酚化合物. 对仲胺而言, 当氮原子上的取代基为脂肪烃或脂环烃 时, 空间结构和核电属性对反应的影响不大(表 3, Entries 1 5), 而且取代基的空间结构越大, 越有利于产物
的结晶; 当氮原子上的取代基为苯环或芐基时，其数量 和空间结构对反应的影响较大(表 3, Entries 6 9), 当氮 原子上的取代基为两个苯环和两个茮基时，均不能得到 相应的产物; 当底物为杂环化合物时, 空间结构是影响 反应的主要因素(表 3 , Entries 10 12). 对苯酚和苯硫酚 而言, 苯硫酚的产率要优于苯酚(表 3, Entries 15，19), 苯环上取代基的种类和数目对反应的影响较大(表 3 , Entries 14 19).

\section{4 催化剂重复使用性能}

以吗啡啉与邻溴异硒氧酸酯为反应模板, 考察碳负 载铜催化剂的重复使用性能, 结果如表 4 所示. 从表 4 可以看出，催化剂经过 4 次使用后，转化效率大约降低 了 $10 \%$ 左右, 但依然具有较好的催化活性. 催化剂活性 下降可能与催化剂多次使用后活性成分少量流失有关.

表 4 催化剂的重复使用性能

Table 4 The performance of $\mathrm{Cu}-\mathrm{C}$ catalyst for repeated use

\begin{tabular}{clcc}
\hline No & Cycle & Reaction time $/ \mathrm{h}$ & Yield/\% \\
\hline 1 & 1st & 1.0 & 82 \\
2 & 2nd & 1.0 & 78 \\
3 & 3rd & 1.2 & 75 \\
4 & 4th & 1.2 & 74 \\
\hline
\end{tabular}

${ }^{a}$ Reaction conditions:formamides $(3 \mathrm{mmol})$, triethylamine $(12.8 \mathrm{mmol})$, triphosgene (1.6 mmol), DBU (3 mmol), secondary amine $(3.6 \mathrm{mmol}), \mathrm{Cu}-\mathrm{C}$ $(0.05 \mathrm{~g}) .{ }^{b}$ Isolated yield based on aryl amines.

\section{2 结论}

报道了一种简便、高效地构建苯并硒唑类衍生物的 催化体系. 该体系以经济实用的碳负载纳米铜作为催化 剂, 采用一锅法, 以较好收率生成相应产物, 具有良好 的普适性，因此该体系非常适用于合成生物学上含有苯 并硒唑框架的重要化合物. 与文献的分步合成相比, 合 成效率更高. 另外, 所制备的新型负载型催化剂, 与文 献所用的磑化亚铜催化剂相比, 更容易回收利用, 后处 理更加方便.

\section{3 实验部分}

\section{1 仪器与试剂}

${ }^{1} \mathrm{H}$ NMR 和 ${ }^{13} \mathrm{C}$ NMR 用 Bruker DRX-500 测定, 以 TMS 为内标, $\mathrm{CDCl}_{3}$ 作溶剂; 高分辨质谱使用安捷伦 6520 Q-TOF LC/MS 仪器测定, $\mathrm{CHCl}_{3}$ 作溶剂; 熔点用 WRS-2A 熔点仪测定. 所用的药品和试剂均为市售的 分析纯或化学纯, 除特别说明外, 未经进一步处理.

\section{2 实验方法}

\subsection{1 活性碳负载纳米铜的制备}

在 $25 \mathrm{~mL}$ 的圆底烧瓶中加入 $2.2 \mathrm{~g}$ 硝酸铜和 $100 \mathrm{~mL}$ 蒸馏水, 搅拌至硝酸铜完全溶解, 室温下加入 $5.0 \mathrm{~g}$ 活性 
表 3 苯并硒唑类化合物的合成 ${ }^{a}$

Table 3 Synthesis of benzoselenazoles

\begin{tabular}{|c|c|c|c|c|c|c|c|}
\hline Entry & $\mathrm{NuH}$ & Product & Yield $^{b} / \%$ & Entry & $\mathrm{NuH}$ & Product & Yield $^{b} / \%$ \\
\hline 1 & $\mathrm{Et}_{2} \mathrm{NH}$ & $3 a$ & 80 & 10 & & $\begin{array}{l}\mathrm{Se} \\
\mathbf{3 j}\end{array}$ & 75 \\
\hline 2 & $i-\mathrm{Pr}_{2} \mathrm{NH}$ & $3 b$ & 80 & 11 & & $3 k$ & 75 \\
\hline 3 & & $3 c$ & 80 & 12 & & 31 & 65 \\
\hline 4 & & $3 d$ & 80 & 13 & & $3 n$ & 75 \\
\hline 5 & $\mathrm{Cy}_{2} \mathrm{NH}$ & & 80 & 14 & & 30 & 72 \\
\hline 6 & $\mathrm{MePhNH}$ & $3 f$ & 75 & 15 & & $3 p$ & 69 \\
\hline 7 & $i$-PrPhNH & $3 g$ & 75 & 16 & & $3 q$ & 80 \\
\hline 8 & $\mathrm{Ph}_{2} \mathrm{NH}$ & $3 \mathrm{~h}$ & 0 & 17 & & $3 r$ & 80 \\
\hline 9 & $\mathrm{Bn}_{2} \mathrm{NH}$ & $3 \mathbf{i}$ & 0 & 18 & & $3 s$ & 78 \\
\hline
\end{tabular}

${ }^{a}$ Reaction conditions: formamides ( $\left.3 \mathrm{mmol}\right)$, triethylamine (12.8 mmol), $\mathrm{CH}_{2} \mathrm{Cl}_{2}(12 \mathrm{~mL})$, triphosgene (1.6 mmol), DBU (3 mmol), THF $(9 \mathrm{~mL})$, secondary amine $(3.6 \mathrm{mmol}), \mathrm{Cu}-\mathrm{C}(0.05 \mathrm{~g}){ }^{b}$ Isolated yield based on aryl amines.

碳后继续摚拌 $0.5 \mathrm{~h}$, 量取 $2.2 \mathrm{~mL} 80 \%$ 水合肼溶于 $15 \mathrm{~mL}$ 的蒸馏水中, 然后缓慢滴入上述混合物中, 加完后继续 搅拌 $1 \mathrm{~h}$, 接着过滤、洗涤, 然后真空抽干滤渣, 即可得 到活性碳负载纳米铜催化剂.

\section{2 .2 苯并硒唑类化合物的合成}

在研钵中加入 $4.0 \mathrm{mmol}$ 邻溴苯胺, $2.0 \mathrm{mmol}$ 氧化锌 和 $6.0 \mathrm{mmol}$ 的甲酸, 用力研磨 $1 \mathrm{~h}$, 而后整体转移到 25 $\mathrm{mL}$ 的三口烧瓶中, 再加入 $1.8 \mathrm{~mL}$ 三乙胺、 $4 \AA$ 分子篮 
和 $8 \mathrm{~mL}$ 二氯甲烷, 加热回流. 将 $1.6 \mathrm{mmol}$ 三光气溶于 4 $\mathrm{mL}$ 二氯甲烷, 缓慢滴入上述溶液中, 滴完后继续反应 $2.5 \mathrm{~h}$, 再加入 $6 \mathrm{mmol}$ 硒粉, 继续反应 $5 \mathrm{~h}$. 反应毕, 过滤 得红褐色溶液, 而后加入 $3 \mathrm{mmol} 1$,8-二氮杂二环十一碳7-烯 $(\mathrm{DBU}) 、 2 \mathrm{~mL}$ 四氢呋喃、3.6 $\mathrm{mmol}$ 仲胺化合物和 $0.05 \mathrm{~g}$ 活性碳负载纳米铜, 室温下搅拌 1 4 h (TLC 跟 踪反应). 反应结束后, 将混合物倒入饱和碳酸氢钠溶 液中, 用乙醚萃取, 有机层用无水硫酸镁干燥, 过滤, 粗产物经柱层析 $[V$ (石油醚) $: V$ (乙酸乙酯 $)=8: 1]$ 分 离, 可得纯化产物, 计算产率. 目标产物通过 ${ }^{1} \mathrm{H}$ NMR、 ${ }^{13} \mathrm{C} N M R$ 和 HRMS 进行结构表征, 其中 $3 \mathbf{d} \sim \mathbf{3 g}, \mathbf{3 k}, \mathbf{3 n}$, $3 p$ 为新化合物.

2-二乙基氨基-1,3-苯并硒唑(3a): 黄色液体, 产率 80\%. ${ }^{1} \mathrm{H}$ NMR (500 MHz, $\left.\mathrm{CDCl}_{3}\right) \delta: 7.57$ (dd, $J=36.0,7.9$ $\mathrm{Hz}, 1 \mathrm{H}), 7.38 \sim 7.18(\mathrm{~m}, 1 \mathrm{H}), 6.95(\mathrm{dd}, J=10.8,4.3 \mathrm{~Hz}$, $1 \mathrm{H}), 3.54$ (q, $J=7.0 \mathrm{~Hz}, 2 \mathrm{H}), 1.28$ (t, $J=7.1 \mathrm{~Hz}, 3 \mathrm{H}$ ); MS (ESI) $m / z: 254.1[\mathrm{M}+\mathrm{H}]^{+}$.

2-二异丙基氨基-1,3-苯并硒唑(3b): 白色固体, 产 率 $80 \% .{ }^{1} \mathrm{H}$ NMR $\left(500 \mathrm{MHz}, \mathrm{CDCl}_{3}\right) \delta: 7.61(\mathrm{~d}, J=7.8$ $\mathrm{Hz}, 1 \mathrm{H}), 7.51(\mathrm{~d}, J=7.9 \mathrm{~Hz}, 1 \mathrm{H}), 7.29 \sim 7.19(\mathrm{~m}, 1 \mathrm{H})$, $6.93(\mathrm{t}, J=7.5 \mathrm{~Hz}, 1 \mathrm{H}), 3.88 \sim 3.70(\mathrm{~m}, 2 \mathrm{H}), 1.44(\mathrm{~d}, J=$ $6.7 \mathrm{~Hz}, 12 \mathrm{H})$; MS (ESI) $m / z: 282.3[\mathrm{M}+\mathrm{H}]^{+}$.

2-(1,4-氧氮杂环己基)-1,3-苯并硒唑(3c): 黄色晶体, 产率 $80 \%$. m.p. $115.5 \sim 116.7{ }^{\circ} \mathrm{C} ;{ }^{1} \mathrm{H}$ NMR $(500 \mathrm{MHz}$, $\left.\mathrm{CDCl}_{3}\right) \delta: 7.68(\mathrm{dd}, J=7.8,0.9 \mathrm{~Hz}, 1 \mathrm{H}), 7.61(\mathrm{~d}, J=8.0$ $\mathrm{Hz}, 1 \mathrm{H}), 7.37 \sim 7.31(\mathrm{~m}, 1 \mathrm{H}), 7.10 \sim 7.01(\mathrm{~m}, 1 \mathrm{H}), 3.90 \sim$ $3.81(\mathrm{~m}, 4 \mathrm{H}), 3.67 \sim 3.60(\mathrm{~m}, 4 \mathrm{H}) ;{ }^{13} \mathrm{C}$ NMR $(125 \mathrm{MHz}$, $\left.\mathrm{CDCl}_{3}\right) \delta: 169.67,154.27,133.36,126.39,124.02,121.81$, 120.65, 66.34, 49.70; MS (ESI) $m / z: 268.2[\mathrm{M}+\mathrm{H}]^{+}$.

2-(1-氮杂环戊基)-1,3-苯并硒唑(3d): 黄色晶体, 产 率 80\%. m.p. 107.2 108.2 ${ }^{\circ} \mathrm{C} ;{ }^{1} \mathrm{H}$ NMR $(500 \mathrm{MHz}$, $\left.\mathrm{CDCl}_{3}\right) \delta: 7.58$ (ddd, $J=9.1,8.0,0.8 \mathrm{~Hz}, 1 \mathrm{H}$ ), 7.23 (ddd, $J=8.1,7.4,1.3 \mathrm{~Hz}, 1 \mathrm{H}), 6.93(\mathrm{td}, J=7.8,1.2 \mathrm{~Hz}, 1 \mathrm{H})$, $3.53(\mathrm{t}, J=6.6 \mathrm{~Hz}, 2 \mathrm{H}), 2.07 \sim 1.97(\mathrm{~m}, 2 \mathrm{H}) ;{ }^{13} \mathrm{C} \mathrm{NMR}$ $\left(125 \mathrm{MHz}, \mathrm{CDCl}_{3}\right) \delta: 169.67,154.27,133.36,126.39$, 124.02, 121.81, 120.65, 66.34, 49.70. HRMS (ESI) calcd for $\mathrm{C}_{11} \mathrm{H}_{13} \mathrm{~N}_{2} \mathrm{Se}[\mathrm{M}+1]^{+}$253.0166, found 253.0112.

2-二环已基氨基-1,3-苯并硒唑(3e): 白色固体, 产 率 $80 \%$. m.p. $103.8 \sim 106.2{ }^{\circ} \mathrm{C} ;{ }^{1} \mathrm{H}$ NMR $(500 \mathrm{MHz}$, $\left.\mathrm{CDCl}_{3}\right) \delta: 7.65(\mathrm{~d}, J=7.7 \mathrm{~Hz}, 1 \mathrm{H}), 7.55(\mathrm{~d}, J=8.0 \mathrm{~Hz}$, $1 \mathrm{H}), 7.28(\mathrm{dd}, J=8.4,6.9 \mathrm{~Hz}, 1 \mathrm{H}), 6.97(\mathrm{t}, J=7.5 \mathrm{~Hz}$, 1H), 3.33 (ddd, $J=11.8,8.5,3.6 \mathrm{~Hz}, 2 \mathrm{H}), 2.23$ (dd, $J=$ 23.0, $11.3 \mathrm{~Hz}, 4 \mathrm{H}), 1.91$ (d, $J=13.2 \mathrm{~Hz}, 4 \mathrm{H}), 1.79$ (d, $J=$ $11.5 \mathrm{~Hz}, 4 \mathrm{H}), 1.73(\mathrm{~d}, J=12.8 \mathrm{~Hz}, 2 \mathrm{H}), 1.46 \sim 1.34(\mathrm{~m}$, $4 \mathrm{H}), 1.33 \sim 1.20(\mathrm{~m}, 2 \mathrm{H}),{ }^{13} \mathrm{C}$ NMR $\left(125 \mathrm{MHz}, \mathrm{CDCl}_{3}\right) \delta$ :
$165.82,155.28,132.71,125.85,123.38,120.55,119.88$, $62.45,30.54,26.41,25.42$. HRMS (ESI) calcd for $\mathrm{C}_{19} \mathrm{H}_{27} \mathrm{~N}_{2} \mathrm{Se} \quad[\mathrm{M}+1]^{+} 363.1360$, found 363.1461 .

2-甲基苯基氨基-1,3-苯并硒唑(3f)：黄色晶体，产率 75\%. m.p.112.2 114.0 ${ }^{\circ} \mathrm{C} ;{ }^{1} \mathrm{H}$ NMR (500 MHz, $\mathrm{CDCl}_{3}$ ) $\delta: 7.66(\mathrm{~d}, J=8.0 \mathrm{~Hz}, 1 \mathrm{H}), 7.56(\mathrm{~d}, J=7.8 \mathrm{~Hz}, 1 \mathrm{H}), 7.53 \sim$ $7.43(\mathrm{~m}, 4 \mathrm{H}), 7.42 \sim 7.36(\mathrm{~m}, 1 \mathrm{H}), 7.35 \sim 7.30(\mathrm{~m}, 1 \mathrm{H})$, $7.08 \sim 7.01(\mathrm{~m}, 1 \mathrm{H}), 3.68(\mathrm{~s}, 3 \mathrm{H}) .{ }^{13} \mathrm{C}$ NMR $(125 \mathrm{MHz}$, $\left.\mathrm{CDCl}_{3}\right) \delta: 169.07,146.92,130.12,127.79,126.20,126.01$, $123.73,122.04,120.44,40.60$. HRMS (ESI) calcd for $\mathrm{C}_{14} \mathrm{H}_{13} \mathrm{~N}_{2} \mathrm{Se}[\mathrm{M}+1]^{+}$289.0166, found 289.0221.

2-异丙基苯基氨基-1,3-苯并硒唑(3g)：黄色晶体, 产率 75\%. m.p.162.7 163.3 ${ }^{\circ} \mathrm{C} ;{ }^{1} \mathrm{H}$ NMR $(500 \mathrm{MHz}$, $\left.\mathrm{CDCl}_{3}\right) \delta$ : $7.64(\mathrm{~s}, 1 \mathrm{H}), 7.57 \sim 7.48(\mathrm{~m}, 4 \mathrm{H}), 7.35(\mathrm{~s}, 3 \mathrm{H})$, $6.99(\mathrm{t}, J=7.5 \mathrm{~Hz}, 1 \mathrm{H}), 1.29(\mathrm{~d}, J=6.7 \mathrm{~Hz}, 6 \mathrm{H}) ;{ }^{13} \mathrm{C} \mathrm{NMR}$ $\left(125 \mathrm{MHz}, \mathrm{CDCl}_{3}\right) \delta: 170.19,154.17,141.88,130.73$, 130.04, 129.24, 126.20, 123.56, 121.67, 120.13, 114.97, 51.71, 21.23. HRMS (ESI) calcd for $\mathrm{C}_{16} \mathrm{H}_{17} \mathrm{~N}_{2} \mathrm{Se}[\mathrm{M}+1]^{+}$ 317.0479 , found 317.0311 .

2-(1-咪唑基)-1,3-苯并硒唑 (3j): 橙色晶体, 产率 75\%. m.p. $125.4 \sim 127.8{ }^{\circ} \mathrm{C} ;{ }^{1} \mathrm{H}$ NMR $\left(300 \mathrm{MHz}, \mathrm{CDCl}_{3}\right.$ ) $\delta: 8.19$ (s, 1H), 7.90 (d, $J=8.1 \mathrm{~Hz}, 1 \mathrm{H}), 7.79$ (d, $J=7.9$ $\mathrm{Hz}, 1 \mathrm{H}), 7.53$ (s, 1H), 7.45 (dd, $J=11.3,4.1 \mathrm{~Hz}, 1 \mathrm{H}), 7.27$ (dd, $J=11.1,4.2 \mathrm{~Hz}, 1 \mathrm{H}), 7.15$ (s, 1H); ${ }^{13} \mathrm{C}$ NMR $(125$ $\left.\mathrm{MHz}, \mathrm{CDCl}_{3}\right) \delta: 156.83,151.86,136.35,135.85,131.25$, $127.19,125.65,124.66,124.39,118.40$; MS (ESI) $\mathrm{m} / \mathrm{z}$ : $249.2[\mathrm{M}+\mathrm{H}]^{+}$.

2-[1-(2-甲基咪唑基)]-1,3-苯并硒唑(3k): 黄色晶体, 产率 75\%. m.p. 47.6 49.9 ${ }^{\circ} \mathrm{C} ;{ }^{1} \mathrm{H}$ NMR $(500 \mathrm{MHz}$, $\left.\mathrm{CDCl}_{3}\right) \delta: 8.02(\mathrm{~d}, J=8.1 \mathrm{~Hz}, 1 \mathrm{H}), 7.89(\mathrm{~d}, J=7.9 \mathrm{~Hz}$, $1 \mathrm{H}), 7.57 \sim 7.49(\mathrm{~m}, 1 \mathrm{H}), 7.42(\mathrm{~d}, J=1.5 \mathrm{~Hz}, 1 \mathrm{H}), 7.38 \sim$ $7.33(\mathrm{~m}, 1 \mathrm{H}), 7.06(\mathrm{~d}, J=1.5 \mathrm{~Hz}, 1 \mathrm{H}), 2.83(\mathrm{~s}, 3 \mathrm{H}),{ }^{13} \mathrm{C}$ NMR $\left(125 \mathrm{MHz}, \mathrm{CDCl}_{3}\right) \delta: 157.50,152.00,145.83$, 136.96, 128.87, 127.03, 125.56, 124.59, 124.44, 120.18, 16.13. HRMS (ESI) calcd for $\mathrm{C}_{11} \mathrm{H}_{10} \mathrm{~N}_{3} \mathrm{Se}[\mathrm{M}+1]^{+}$ 263.9968, found 264.0112.

2-(1-苯并咪唑基)-1,3-苯并硒唑(31): 白色晶体, 产 率 65\%. ${ }^{1} \mathrm{H}$ NMR $\left(500 \mathrm{MHz}, \mathrm{CDCl}_{3}\right) \delta: 8.56(\mathrm{~s}, 1 \mathrm{H}), 8.36$ (d, $J=7.8 \mathrm{~Hz}, 1 \mathrm{H}), 8.07$ (d, $J=8.0 \mathrm{~Hz}, 1 \mathrm{H}), 7.92$ (t, $J=6.4$ $\mathrm{Hz}, 2 \mathrm{H}), 7.60 \sim 7.43(\mathrm{~m}, 4 \mathrm{H}), 7.37(\mathrm{t}, J=7.6 \mathrm{~Hz}, 1 \mathrm{H}) ; \mathrm{MS}$ (ESI) $m / z: 299.2[\mathrm{M}+\mathrm{H}]^{+}$.

2-苯氧基-1,3-苯并硒唑 $(\mathbf{3 n})$ : 紫色固体, 产率 75\%. ${ }^{1} \mathrm{H}$ NMR $\left(500 \mathrm{MHz}, \mathrm{CDCl}_{3}\right) \delta: 7.80(\mathrm{dd}, J=8.1,0.7 \mathrm{~Hz}$, $1 \mathrm{H}), 7.71(\mathrm{dd}, J=7.9,0.8 \mathrm{~Hz}, 1 \mathrm{H}), 7.49(\mathrm{tt}, J=4.2,2.1 \mathrm{~Hz}$, $2 \mathrm{H}), 7.45 \sim 7.33(\mathrm{~m}, 4 \mathrm{H}), 7.22(\mathrm{td}, J=7.9,1.2 \mathrm{~Hz}, 1 \mathrm{H})$; 
${ }^{13} \mathrm{C}$ NMR $\left(125 \mathrm{MHz}, \mathrm{CDCl}_{3}\right) \delta: 173.58,155.61,150.60$, $135.72,130.12,126.71,126.49,124.50,124.17,123.15$, 120.96. HRMS (ESI) calcd for $\mathrm{C}_{13} \mathrm{H}_{10}$ NOSe $[\mathrm{M}+1]^{+}$ 275.9859, found 275.9950 .

2-(4-氯苯氧基)-1,3-苯并硒唑 $(\mathbf{3 o})$ ：黄色晶体，产率 72\%. m.p. 58.7 61.9 ${ }^{\circ} \mathrm{C} ;{ }^{1} \mathrm{H}$ NMR (500 MHz, $\left.\mathrm{CDCl}_{3}\right) \delta$ : $7.78 \sim 7.73(\mathrm{~m}, 1 \mathrm{H}), 7.70(\mathrm{~d}, J=7.9 \mathrm{~Hz}, 1 \mathrm{H}), 7.44 \sim 7.36$ $(\mathrm{m}, 3 \mathrm{H}), 7.35 \sim 7.30(\mathrm{~m}, 2 \mathrm{H}), 7.24 \sim 7.17(\mathrm{~m}, 1 \mathrm{H}) ;{ }^{13} \mathrm{C}$ NMR (125 MHz, $\left.\mathrm{CDCl}_{3}\right) \delta: 172.71,153.75,150.36$, $135.77,131.88,130.10,126.59,124.57,124.40,123.23$, 122.27; MS (ESI) $m / z: 309.1[\mathrm{M}+\mathrm{H}]^{+}$.

2-(3-甲基-4-氯苯氧基)-1,3-苯并硒唑(3p): 橙色固 体, 产率 69\%. m.p. 72.0 73.2 ${ }^{\circ} \mathrm{C} ;{ }^{1} \mathrm{H}$ NMR $(500 \mathrm{MHz}$, $\left.\mathrm{CDCl}_{3}\right) \delta: 7.74(\mathrm{~d}, J=8.1 \mathrm{~Hz}, 1 \mathrm{H}), 7.65(\mathrm{~d}, J=7.9 \mathrm{~Hz}$, $1 \mathrm{H}), 7.39 \sim 7.31(\mathrm{~m}, 2 \mathrm{H}), 7.22(\mathrm{dd}, J=6.6,1.9 \mathrm{~Hz}, 1 \mathrm{H})$, $7.18 \sim 7.09(\mathrm{~m}, 2 \mathrm{H}), 2.37(\mathrm{~s}, 3 \mathrm{H}) ;{ }^{13} \mathrm{C} \mathrm{NMR}(125 \mathrm{MHz}$, $\left.\mathrm{CDCl}_{3}\right) \delta: 173.06,153.67,150.48,138.21,135.81,132.09$, $130.55,130.34,126.57,124.61,124.35,123.22,119.60$, 20.38. HRMS (ESI) calcd for $\mathrm{C}_{14} \mathrm{H}_{11}$ CINOSe $[\mathrm{M}+1]^{+}$ 323.9618 , found 2323.9778 .

2-(4-甲基苯硫基)-1,3-苯并硒唑(3q)：白色固体，产 率 80\%. m.p. $77.9 \sim 79.4{ }^{\circ} \mathrm{C} ;{ }^{1} \mathrm{H}$ NMR $\left(500 \mathrm{MHz}, \mathrm{CDCl}_{3}\right.$ ) $\delta: 7.91 \sim 7.84(\mathrm{~m}, 1 \mathrm{H}), 7.65(\mathrm{dd}, J=9.9,4.9 \mathrm{~Hz}, 3 \mathrm{H}), 7.37$ (ddd, $J=8.2,7.2,2.9 \mathrm{~Hz}, 1 \mathrm{H}), 7.28(\mathrm{dd}, J=25.5,6.8 \mathrm{~Hz}$, $2 \mathrm{H}), 7.21 \sim 7.14(\mathrm{~m}, 1 \mathrm{H}), 2.44(\mathrm{~d}, J=4.6 \mathrm{~Hz}, 3 \mathrm{H}) ;{ }^{13} \mathrm{C}$ NMR $\left(125 \mathrm{MHz}, \mathrm{CDCl}_{3}\right) \delta: 174.78,155.86,141.57$, $138.44,135.77,130.90,127.74,126.24,124.20,123.97$, 123.23, 21.50; MS (ESI) $m / z: 305.1[\mathrm{M}+\mathrm{H}]^{+}$.

2-(2-甲基苯硫基)-1,3-苯并硒唑(3r): 白色固体, 产 率 80\%. m.p. 83.5 85.4 ${ }^{\circ} \mathrm{C} ;{ }^{1} \mathrm{H}$ NMR (500 MHz, $\mathrm{CDCl}_{3}$ ) $\delta: 7.90(\mathrm{~d}, J=8.1 \mathrm{~Hz}, 1 \mathrm{H}), 7.79$ (d, $J=7.6 \mathrm{~Hz}, 1 \mathrm{H}), 7.69$ $(\mathrm{d}, J=7.9 \mathrm{~Hz}, 1 \mathrm{H}), 7.50(\mathrm{t}, J=7.1 \mathrm{~Hz}, 1 \mathrm{H}), 7.42$ (dd, $J=$ 15.8, 7.7 Hz, 2H), 7.35 (t, $J=7.5 \mathrm{~Hz}, 1 \mathrm{H}), 7.21$ (t, $J=7.5$ $\mathrm{Hz}, 1 \mathrm{H}), 2.58(\mathrm{~s}, 3 \mathrm{H}) ;{ }^{13} \mathrm{C} \mathrm{NMR}\left(125 \mathrm{MHz}, \mathrm{CDCl}_{3}\right) \delta$ : $173.84,155.94,143.45,138.46,137.31,131.62,130.65$, 127.60, 126.25, 124.18, 124.03, 123.23, 20.97; MS (ESI) $m / z: 305.2[\mathrm{M}+\mathrm{H}]^{+}$.

2-(4-氯苯硫基)-1,3-苯并硒唑 $(3 \mathrm{~s})$ ：白色固体，产率
78\%. m.p. 99.2 101.3 ${ }^{\circ} \mathrm{C} ;{ }^{1} \mathrm{H}$ NMR $\left(500 \mathrm{MHz}, \mathrm{CDCl}_{3}\right) \delta$ : $7.91(\mathrm{~d}, J=8.2 \mathrm{~Hz}, 1 \mathrm{H}), 7.73$ (d, $J=8.5 \mathrm{~Hz}, 3 \mathrm{H}), 7.50$ (d, $J=8.1 \mathrm{~Hz}, 2 \mathrm{H}), 7.43(\mathrm{t}, J=7.7 \mathrm{~Hz}, 1 \mathrm{H}), 7.24(\mathrm{t}, J=7.6$ $\mathrm{Hz}, 1 \mathrm{H}) ;{ }^{13} \mathrm{C} \mathrm{NMR}\left(125 \mathrm{MHz}, \mathrm{CDCl}_{3}\right) \delta: 172.12,155.62$, $138.59,137.42,136.83,130.34,129.55,126.41,124.55$, 124.07, 123.47; MS (ESI) $m / z: 325.2[\mathrm{M}+\mathrm{H}]^{+}$.

辅助材料(Supporting Information) 所有新化合物 ${ }^{1} \mathrm{H}$ $\mathrm{NMR}$ 和 ${ }^{13} \mathrm{C} \mathrm{NMR}$ 谱图. 这些材料可以免费从本刊网 站(http://sioc-journal.cn/)上下载.

\section{References}

[1] (a) Mugesh, G. Curr. Chem. Biol. 2013, 7, 47.

(b) Papp, L. V.; Lu, J.; Holmgren, A.; Khanna, K. K. Antioxid. Redox Signaling 2007, 9, 775.

(c) Li, T., Yi, Y., Xu, H. Acta Chim. Sinica 2014, 72, 1079 (in Chinese). (李天予, 易宇, 许华平, 化学学报, 2014, 72, 1079.)

[2] Bayoumy, K., Sinha, R. Mutat. Res., Fundam. Mol. Mech. Mutagen. 2004, 551, 81.

[3] (a) Santos, P. F.; Reis, L. V.; Almeida, P.; Serrano, J. P.; Oliveira, A. S.; Ferreira, L. F. V. J. Photochem. Photobiol. A 2004, 163, 267.

(b) Santos, P. F.; Reis, L. V.; Almeida, P.; Oliveira, A. S.; Ferreira, L. F. V. J. Photochem. Photobiol. A 2003, 160, 159.

[4] (a) Beletskaya, I. P.; Cheprakov, A. V. Coord. Chem. Rev. 2004, $248,2337$.

(b) Ley, S. V.; Thomas, A. W. Angew. Chem., Int. Ed. 2003, 42, 5400 .

(c) Font, M.; Parella, T.; Costas, M.; Ribas, X. Organometallics 2012, 31, 7976.

[5] Fujiwara, S.; Asanuma, Y.; Shin-ike, T.; Kambe, N. J. Org. Chem. 2007, 72, 8087.

[6] (a) Sashida, H.; Nakabayashi, S.; Suzuki, H.; Kaname, M.; Minoura, M. Tetrahedron Lett. 2010, 51, 5395.

(b) Sashida, H. Heterocycles 2011, 83, 2223.

[7] Kaname, M.; Minoura, M.; Sashida, H. Tetrahedron Lett. 2011, 52, 505.

[8] (a) Xu, H.; Man, Q.; Lin, Y.; Li, Y.; Feng, Y. Chin. J. Org. Chem. 2010, 30, 9 (in Chinese).

(许华建, 蔄秋石, 林义成, 李源源, 冯乙巳, 有机化学, 2010, 30, 9.)

(b) Dong, L.; Yao, X.; Chen, Y. Chin. J. Catal. 2013, 34, 851 (in Chinese).

(董林, 姚小江, 陈懿, 催化学报, 2013, 34, 851.)

(c) Ren, J.; Guo, C.; Yang, L.; Li, Z. Chin. J. Catal. 2013, 34, 1734 (in Chinese).

(任军, 郭长江, 杨雷雷, 李忠, 催化学报, 2013, 34, 1734.)

(Qin, X.) 